\title{
An Empirical Analysis on the Effect of OFDM Parameters to the Performance of Wireless Communication System via USRP-based Transceiver
}

\author{
Jusnaini Muslimin ${ }^{1}$, Ani Liza Asnawi ${ }^{1}$, Ahmad Fadzil Ismail ${ }^{1}$, Muhamad Haziq \\ Khairolanuar ${ }^{1}$, Ahmad Zamani Jusoh ${ }^{1}$ and Rosyati Hamid ${ }^{2}$ \\ ${ }^{1}$ Electrical and Computer Engineering Department, Kulliyyah of Engineering \\ International Islamic University Malaysia,Kuala Lumpur, Malaysia \\ ${ }^{2}$ Faculty of Electrical \& Electronics Engineering, University Malaysia Pahang \\ (UMP) \\ jusnainimuslimin@gmail.com,aniliza@iium.edu.my,af_ismail@iium.edu.my, \\ haziq.khairolanuar@yahoo.com,azamani@iium.edu.my,rosyati@ump.edu.my
}

\begin{abstract}
The configuration of parameters for OFDM scheme in standards such as the 802.11, WiMAX and LTE are varied accordingly to each system specifications. This paper offers findings from an experimental analysis carried out using wireless OFDM transceivers set-up. Experimental evaluations are indeed very useful in order to guesstimate the likely performance of any said system. Among particular system performances of interest include throughput and errors. This can be noteworthy attempt as it provides fundamental understanding and knowledge in justifying what should be the best configuration. The study had examined and quantified the effect of varying select parameters namely the modulation schemes, FFT length, and sampling rate. The use of USRP hardware as the communication testbed was extensively explored during the study. USRP sets can certainly be emerging tools for students and researchers alike to realize proof of concepts by exploiting programmable software-defined radio platforms.
\end{abstract}

Keywords: OFDM; USRP; GNU Radio; Wireless Communication; Modulation; FFT; Sampling Rate

\section{Introduction}

The upcoming "Internet-of-Things" (IoT) predicts that 26 billion devices will be interconnected wirelessly by 2020 [1]. As such, study on improving the techniques or mechanisms of current communication method such as the Orthogonal Frequency Division Multiplexing (OFDM) is always of great interest [2]. While studies of OFDM are extensively being explored through simulation approaches [3]-[8], there is certainly limited reports of hardware-based experiments that can be retrieved [9], [10]. An experimentation analysis can be vital as it can provide a realistic references of how the communication systems will behave and perform in real-life operating environment. The traditional way of using embedded system for real experimentation without a doubt can indeed be very expensive and involve painstaking efforts. The present availability of open source software-defined radios (SDR) has offered both low development cost and flexibility to deliver the high reconfigurable requirements of communication system [11], [12]. Universal Software Radio Peripheral (USRP) [13] is a range of software-defined radios that can isolate the baseband processing from the RF parts. This has allowed the

Received (June 13, 2017), Review Result (August 28, 2017), Accepted (September 8, 2017) 
modification of system design to be carried out by changing configuration on software basis while retaining the same set of hardware.

\section{Experimental Setup}

The experiment conducted encompasses of SDR platforms namely two USRPs and the GNU Radio toolkits. The USRPs had been deployed as transmitter and receiver set. The transmitter was assembled using a USRP N210 with mounted CBX-40 daughterboard and connected via Ethernet gigabit cable to an i7 laptop running on Ubuntu 14.04. The GNU Radio version 3.7.9.2 facilitated the signal processing in implementation of software radios. Similar assembly was configured as the receiver with minor modification where a desktop was used instead of a laptop. The overview of the experiment setup is shown as in figure 2 .

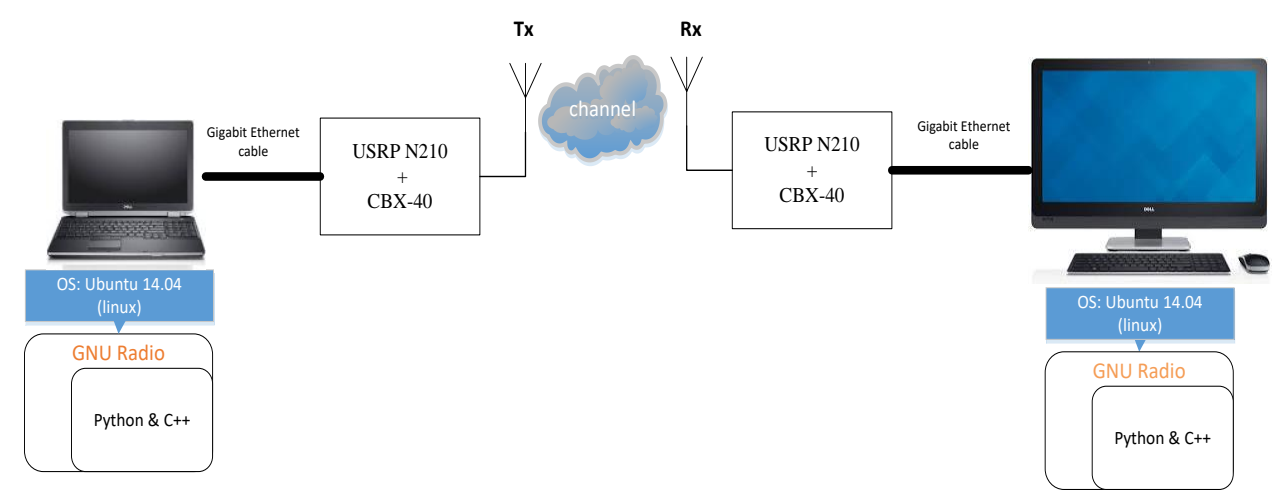

Figure 1. Experimental Setup

The architecture of the OFDM-based transmit and receive is depicted in Figure 3. Monopole antennas with RF center frequency of $2.45 \mathrm{GHz}$ were used. The separation distance between them was set at $66 \mathrm{~cm}$. When using USRP, there is no direct measurement value. The value for most configurable parameters is a relative value. For instance, the value of gain and amplitude can be set to any number in order to figure out the working range of the parameters as well as their corresponding real value, the output power of USRP was measured using CXA Signal Analyzer model N9000A prior to the experimentation. During the experiment, the USRP was connected to the signal analyzer tuned at $2.45 \mathrm{GHz}$.

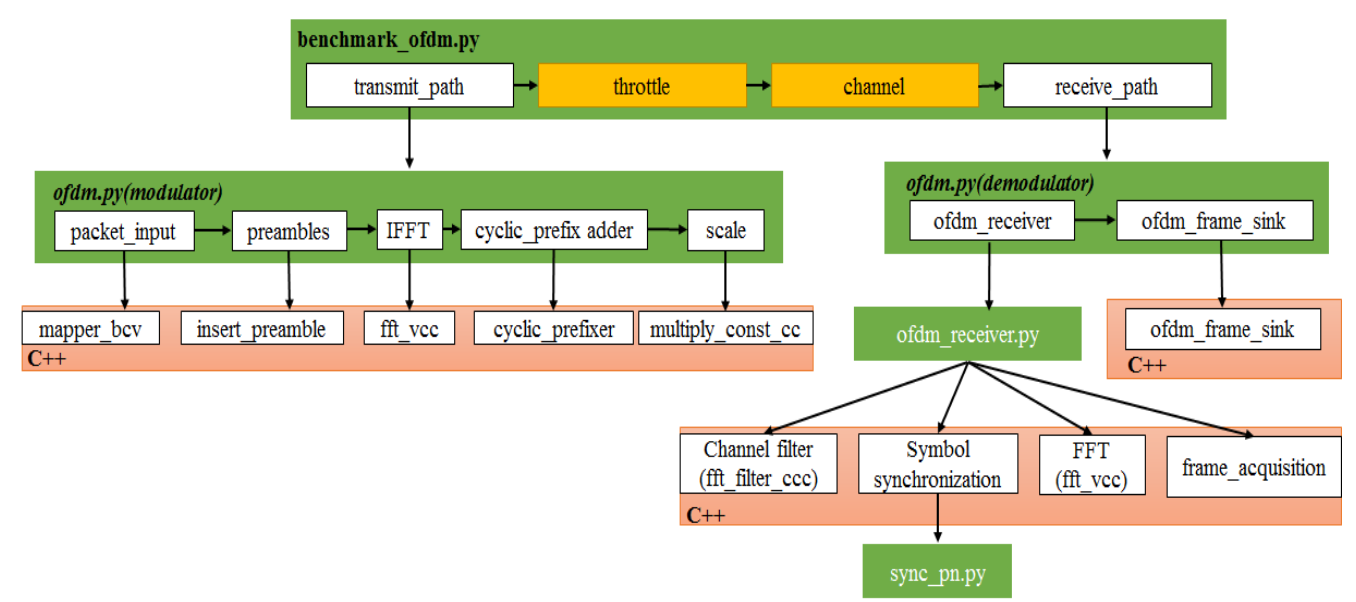

Figure 2. Implemented OFDM Architecture 


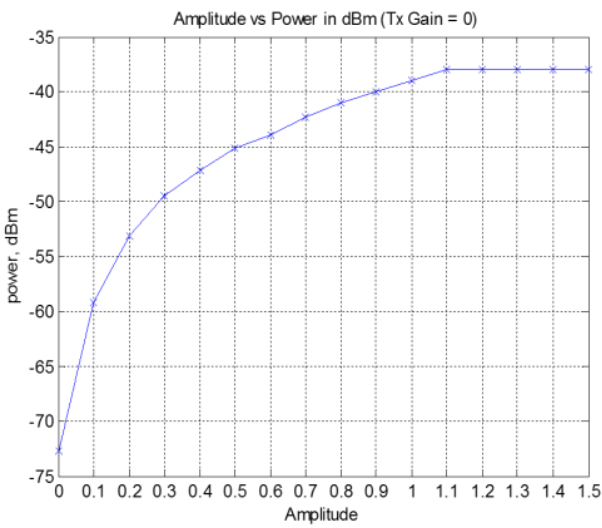

Figure 3. USRP Output Power w.r.t Amplitude Variable

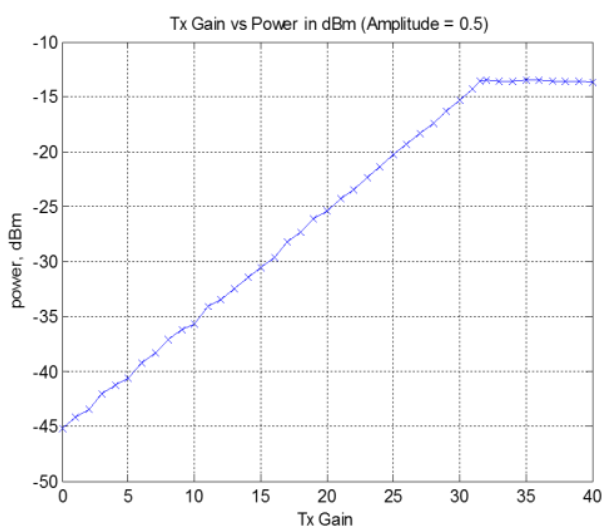

Figure 4. USRP output Power w.r.t Gain Variable

In Figure 4, the associated measured power is shown for amplitude 0 to 1.5 when gain is set to 0 . It can be observed that the power continuously increases until where the amplitude value is about 1.1. For amplitude 1.1 and above, the output power appears to be constant at about $-38 \mathrm{dBm}$. In Figure 5, the measured power is shown for gain 0 to 40 when the amplitude is set at 0.5. From the graph, it can be observed that the power increases around $1 \mathrm{dBm}$ for every gain increment value until gain is 31.5 . This value was regarded as the maximum gain of $\mathrm{CBX}$ daughterboard used in the experiment. Based on the setup, it can be regarded that the working range of the amplitude is from 0 to 1 , while gain is from 0 to 31.5 .

\section{Variation of Parameters}

This section outlines the findings namely change of performance values due to the effect when the OFDM parameters were varied. Three basic OFDM parameters were inspected during study, which are modulation schemes, FFT length and sampling rate. The following graphs present the percentage of either throughput or packet error at the corresponding Rx Gain with respect to the evaluated parameters:

\subsection{Modulations}

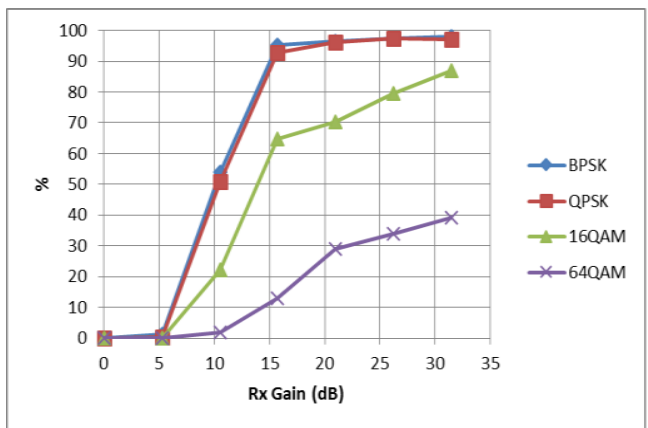

Figure 5. Throughput of Different Modulation Schemes

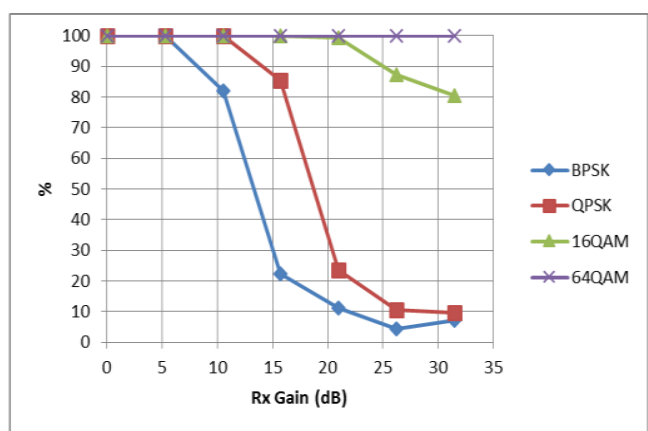

Figure 6. Packet Error of Different Modulation Schemes

Four modulation schemes; BPSK, QPSK, 16QAM, and 64QAM were investigated. Figure 6 and 7 represent the throughput and packet error for each of the modulation as the receiver gain was increased from 0 to 31.5. It can be observed from Figure 6 that the higher the gain, the higher the throughput. It is evident as shown in Figure 7 that the 
packet error reduces as gain is increased for all the modulation schemes except for 64QAM. It appeared as if all the packets experienced error.

The experimentation recorded that lower order modulation scheme of PSK shows better performance than that of QAM modulation scheme. It can be viewed that the throughput of BPSK and QPSK are almost the same. As for QAM, all the received packets appeared to be corrupted for the64QAM scheme. The graph portrays that the 16QAM outperforms the throughput of 64QAM at maximum Rx gain value. 16QAM also experienced reduced packet error of around $80 \%$

\subsection{FFT Length}

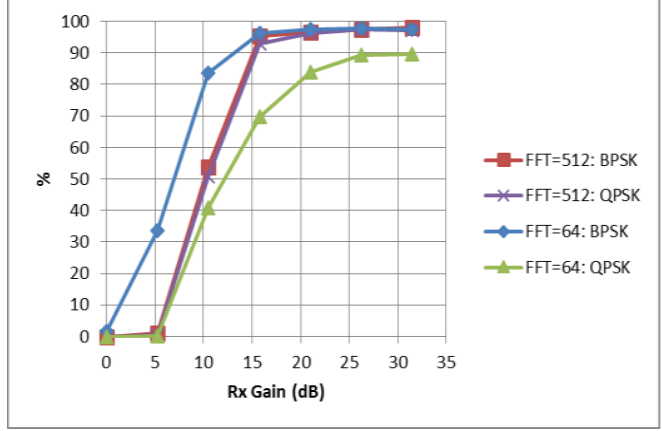

Figure 7. Throughput of PSK in Different FFT Length

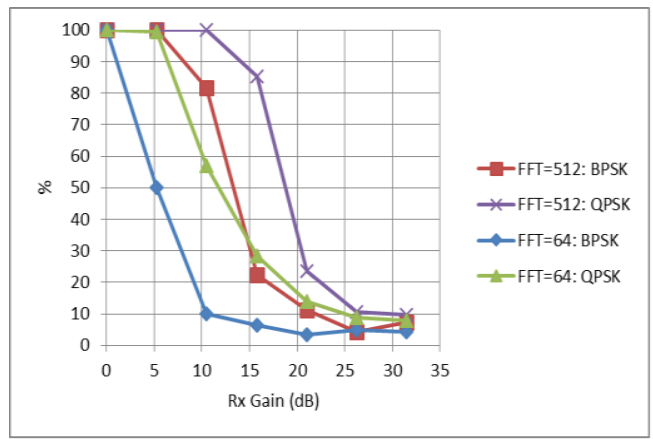

Figure 9. Packet Error of PSK in Different FFT Length

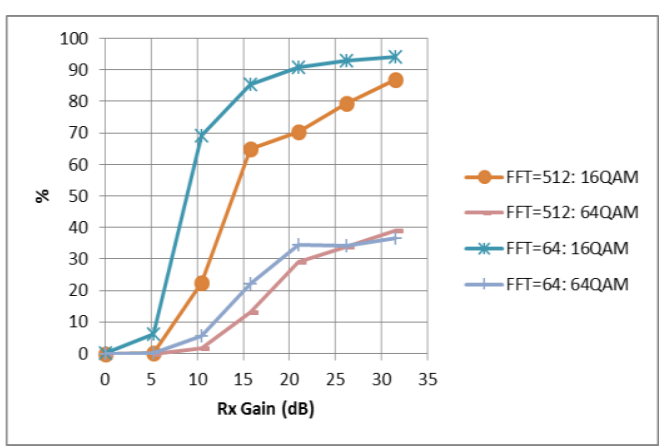

Figure 8. Throughput of QAM in Different FFT Length

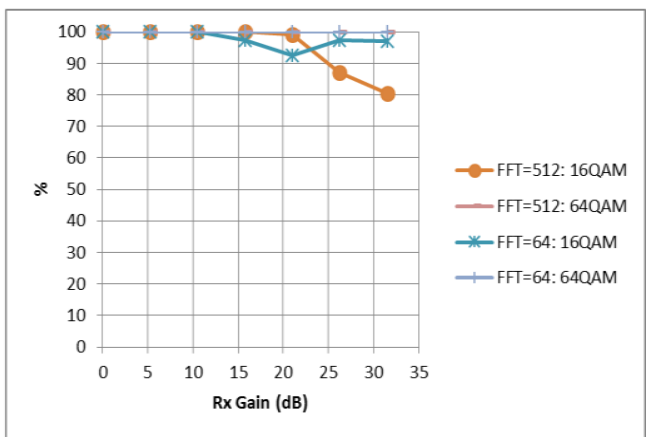

Figure 10. Packet Error of QAM in Different FFT Length

Two FFT lengths were chosen is this study, which are 64 and 512. These FFT lengths were put into test with four modulation techniques, which are BPSK, QPSK, 16QAM and 64QAM. Again the analyses were made in terms of throughput and packet error. Figure 8 shows the throughput for BPSK and QPSK modulation techniques when the FFT lengths were varied. From the observation of Figure 8, it can be identified that BPSK with 64 FFT length exhibits higher throughput for Rx gain above $0 \mathrm{~dB}$. Figure 10 shows the packet error for BPSK and QPSK modulation techniques with different FFT lengths. From the observation of Figure 10, it can be recognized that BPSK with 64 FFT length shows the lowest packet error for $\mathrm{Rx}$ gain above $0 \mathrm{~dB}$. Figure 9 illustrates the throughput measurements for 16QAM and 64QAM modulation techniques. From the observation of Figure 9, it can be stated that 16QAM with 64 FFT length outperforms the 64QAM. The packet error for 16QAM and 64QAM modulation techniques with different FFT lengths can be observed in Figure 11. It can be inferred that all FFT lengths with QAM modulation exhibits packet error at $100 \%$ for values of $10 \mathrm{~dB} \mathrm{Rx}$ gain and below. For $\mathrm{Rx}$ gains between $10 \mathrm{~dB}$ until $22 \mathrm{~dB}, 16 \mathrm{QAM}$ modulation techniques with FFT length 64 shows a slightly lower packet error when compare with the rest. For Rx gain above $22 \mathrm{~dB}$, 
the 16QAM modulation technique with FFT length 512 points out that it has the lowest packet error.

\subsection{Sampling Rate}

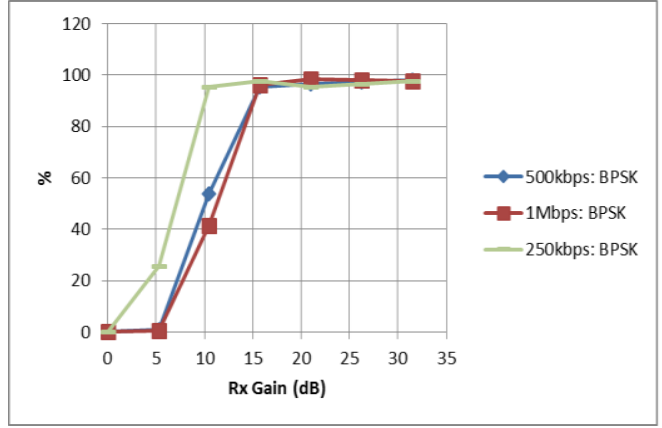

Figure 11. Throughput of BPSK in Different Sampling Rate

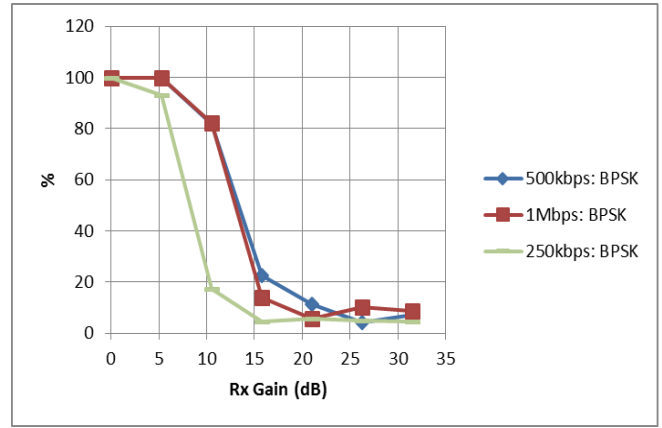

\section{Figure 13. Packet Error of BPSK in Different Sampling Rate}

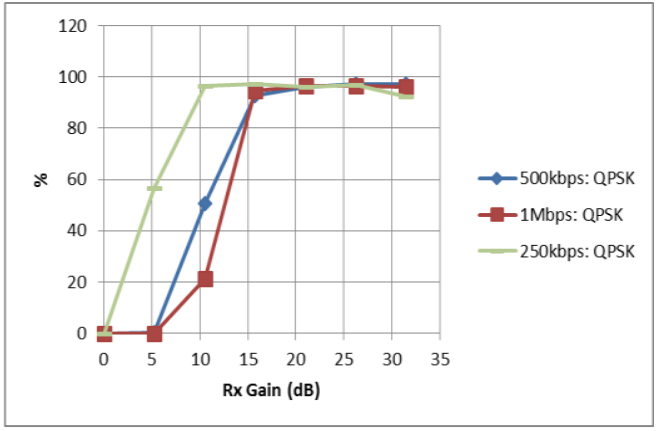

Figure 12. Throughput of QPSK in Different Sampling Rate

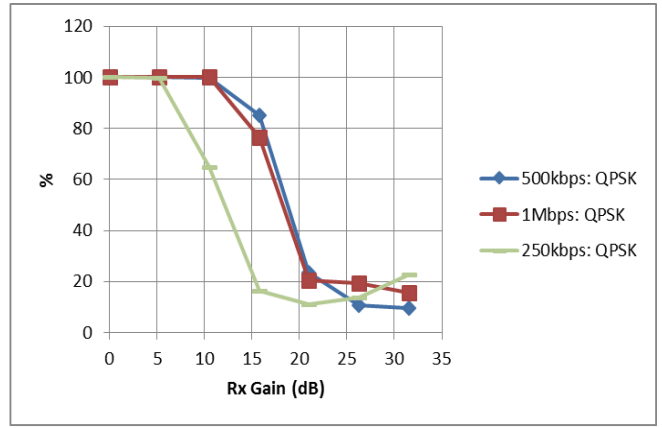

Figure 14. Packet Error of QPSK in Different Sampling Rate

Three sampling rate at $250 \mathrm{kbps}, 500 \mathrm{kpbs}$ and $1 \mathrm{Mbps}$ were chosen in the study. The sampling rates are put into test when using two different types of modulation, which are BPSK and QPSK. From the observation of Figure 12, it can be stated that BPSK with 250 kbps has better throughput compare to the others for Rx gain values between $0 \mathrm{~dB}$ and 15 $\mathrm{dB}$. For Rx gain above $15 \mathrm{~dB}$, all sampling rates have maximum throughput at $100 \%$. The throughput for QPSK modulation techniques with different sampling rate is plotted in Figure 13. For Rx gain values from $0 \mathrm{~dB}$ until $15 \mathrm{~dB}$, it can be recognized that BPSK with $250 \mathrm{kbps}$ has the best. All sampling rates appear to achieve maximum throughput for $\mathrm{Rx}$ gain above $15 \mathrm{~dB}$,

Figure 14 on the other hand represents the packet error for BPSK modulation techniques with different sampling rate. For Rx gain values below $20 \mathrm{~dB}$, it can be observed that BPSK with $250 \mathrm{kbps}$ has lowest packet error compare to the rest. For Rx gain above $20 \mathrm{~dB}$, all sampling rates show minimum packet error below $20 \%$. The packet error for QPSK modulation techniques is illustrated in Figure 15. It was discovered that QPSK with $250 \mathrm{kbps}$ has lowest packet error compare for Rx gain values below $25 \mathrm{~dB}$. For Rx gain above $25 \mathrm{~dB}$, all sampling rates show minimum packet error below $22 \%$. 


\section{Comparison Analysis between Parameters}

The experimental results of each tested parameters were scrutinized in order to identify how significant their effect to the system performance. For this reason, the average rootmean squared error (RMSE) and coefficient of variations of the outputs were calculated and used to deduce the aforementioned level of significance. The RMSE outlines how much the findings deviate from the ideal values which are 100\% throughput and 0\% error. The RMSE in this study is defined as:

$$
\text { RMSE }=\sqrt{\frac{1}{n} \sum_{i=1}^{n}\left(\text { Theory }_{i}-\text { Experiment }_{i}\right)^{2}}
$$

where $n$ is the number of data, Theory is the theoretical data and Experiment is the measurement data. The low RMSE value is desirable as it reflects the result is closer to the maximum throughput and minimum packet error.

Table 1. RMSE of Modulation Scheme

\begin{tabular}{|c|c|c|}
\hline & $\begin{array}{c}\text { RMSE: } \\
\text { Throughput }(\boldsymbol{\%})\end{array}$ & $\begin{array}{c}\text { RMSE: } \\
\text { Packet Error (\%) }\end{array}$ \\
\hline BPSK & 56.00016 & 62.56392 \\
\hline QPSK & 56.59579 & 73.69532 \\
\hline 16QAM & 64.06329 & 95.53628 \\
\hline 64QAM & 84.80045 & 100 \\
\hline
\end{tabular}

Table 2. RMSE of FFT Length

\begin{tabular}{|c|c|c|c|c|}
\hline Modulation & \multicolumn{2}{|c|}{ RMSE: Throughput (\%) } & \multicolumn{2}{c|}{ RMSE: Packet Error (\%) } \\
\hline & FFT = 64 & FFT = 512 & FFT = 64 & FFT = 512 \\
\hline BPSK & 45.35179 & 56.00016 & 42.62132 & 62.56392 \\
\hline QPSK & 59.58964 & 56.59579 & 58.93952 & 73.69532 \\
\hline 16QAM & 53.6692 & 64.06329 & 97.81962 & 95.53628 \\
\hline 64QAM & 82.47248 & 84.80045 & 100 & 100 \\
\hline
\end{tabular}

Table 3. RMSE of Sampling Rate

\begin{tabular}{|c|c|c|c|c|}
\hline Sampling Rate & \multicolumn{2}{|c|}{ RMSE: Throughput (\%) } & \multicolumn{2}{c|}{ RMSE: Packet Error (\%) } \\
& BPSK & QPSK & BPSK & QPSK \\
\hline & 45.35179 & 56.00016 & 42.62132 & 62.56392 \\
\hline 250kbps & 59.58964 & 56.59579 & 58.93952 & 73.69532 \\
\hline 1Mbpps & 53.6692 & 64.06329 & 97.81962 & 95.53628 \\
\hline
\end{tabular}

Table 1-3 depict the RMSE of the system performance. From the analysis, it can be identified that the best performance of the tested configurations was achieved when the FFT length of OFDM was set to 64 with BPSK modulation. From the analysis it can be inferred that 64QAM might not be a reliable option to be deployed since it exhibited total packet error. 
Table 4. Coefficient of Variations for Comparison Between Parameters

\begin{tabular}{|c|c|c|}
\hline \multicolumn{3}{|c|}{ Coefficient of Variation (\%) } \\
\hline & Throughput & Packet Error \\
\hline Modulation Scheme & 20.60185 & 21.45717 \\
\hline FFT Length & 8.238996 & 11.05404 \\
\hline Sampling Rate & 15.1232 & 10.9803 \\
\hline
\end{tabular}

Coefficients of variations are used as the markers for parameters comparison. The coefficient were computed by dividing the standard deviation of each parameters to its corresponding average. The higher the coefficient variation, the more susceptible the system to the changes of tested parameters. From Table 4, modulation scheme gives the highest variation followed by sampling rate and finally FFT length. In other words, modulation scheme is the most significant parameter to the performance of the tested wireless system.

\section{Conclusion}

The findings present prospect of hardware platforms in the assembly of OFDM systems. The setup can incorporate multipath propagation model as a separate system or as a single system readied for analysis. The acquired digital constellation plots and FFT spectrums match the expected theoretical results. Therefore, the study concluded that SDR can be exploited to model OFDM scheme in applications such as WiFi, WiMAX and others. It is noted that although all the parameters in the experiment were set prior to the system execution, the results comply with the theoretical trends. The results suggest that modulation scheme should take priority over other parameters since it dominates the system performance. In a nutshell, appropriate modulation scheme must be on the top consideration followed by other parameters when designing the best OFDM scheme for wireless system.

\section{Acknowledgements}

The authors acknowledge the Research Management Centre of the International Islamic University Malaysia (IIUM) and Malaysian Ministry of Higher Education for the financial assistance. The reported research outcomes are part of the deliverables for the funded research.

\section{References}

[1] J. Gubbi, R. Buyya, S. Marusic and M. Palaniswami, "Internet of Things (IoT): A vision, architectural elements, and future directions”, Futur. Gener. Comput. Syst., vol. 29, no. 7, (2013), pp. 1645-1660.

[2] T. H. T. Hwang, C. Y. C. Yang, G. W. G. Wu, S. L. S. Li and G. Y. Li, "OFDM and Its Wireless Applications: A Survey", IEEE Trans. Veh. Technol., vol. 58, (2009).

[3] L. Wan, H. Zhou, X. Xu, Y. Huang, S. Zhou, Z. Shi and J. H. Cui, "Adaptive Modulation and Coding for Underwater Acoustic OFDM”, IEEE J. Ocean. Eng., vol. 40, no. 2, (2015), pp. 327-336.

[4] G. Lim, S. K. Wilson, L. J. Cimini and D. P. Taylor, "On higher order modulations for OFDM in frequency-selective fading channels”, IEEE Commun. Lett., vol. 17, no. 4, (2013), pp. 641-644.

[5] Y. F. Liu, C. H. Yeh, C. W. Chow and Y. Liu, "Adaptive control of OFDM data rate for LED visible light communications in different free space transmission distances and offsets", Commun. Photonics Conf. (ACP), 2012 Asia, (2012), pp. 1-3.

[6] S. Sun, Y. Ju and Y. Yamao, "Overlay cognitive radio OFDM system for 4G cellular networks", IEEE Wirel. Commun., vol. 20, no. 2, (2013), pp. 68-73.

[7] T. Liu, N. Cao, M. Mao, F. Wang and Y. Du, "Waveform design and optimization for OFDM radar signal based on fractional Fourier transform", in The 2014 2nd International Conference on Systems and Informatics (ICSAI 2014), (2014), pp. 724-729.

[8] S. H. O. Salih, A. Al-Refai, M. Suliman and A. Mohammed, "Implementation of adaptive modulation for broadband wireless access networks using cognitive radio approaches", 2013 Int. Conf. Comput. Electr. Electron. Eng., (2013), pp. 345-349. 
[9] T. Vilches and D. Dujovne, "GNUradio and 802.11: Performance evaluation and limitations," IEEE Netw., vol. 28, no. 5, (2014), pp. 27-31.

[10] B. Bloessl, M. Segata, C. Sommer and F. Dressler, “An IEEE 802 . 11a / g / p OFDM Receiver for GNU Radio", in 2nd ACM SIGCOMM Workshop of Software Radio Implementation Forum (SRIF 2013), (2013).

[11] T. Ulversoy, T. Ulversøy, A. Software and R. Sdr, "Software defined radio: Challenges and opportunities", IEEE Commun. Surv. Tuts., vol. 12, no. 4, (2010), pp. 531-550.

[12] J. M. Mitola, "Software Radios Survey, Critical Evaluation and Future Directions", IEEE Aerosp. Electron. Syst. Mag., vol. 8, no. 4, (1993), pp. 25-36.

[13] M. Ettus and B. Martin, "The Universal Software Radio Peripheral (USRP) Family of Low-Cost SDRs", in Opportunistic Spectrum Sharing and White Space Access: The Practical Reality, O. Holland, H. Bogucka, and A. Medeisis, Eds. Hoboken, NJ: John Wiley \& Sons, (2015), pp. 3-23.

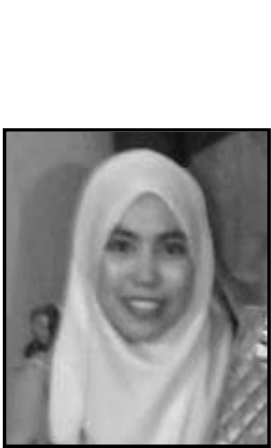

\section{Authors}

J. Muslimin, he received the B.Eng. degree in ElectronicsComputer and Information from the International Islamic University Malaysia (IIUM) in 2011. Currently, she is pursuing the M.S. degree with the Department of Electrical and Computer Engineering, IIUM, in software-defined radio platform research. Her research interests include OFDM and wireless communication systems.

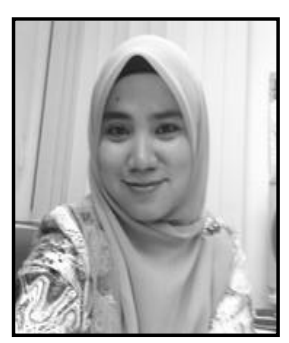

A.L. Asnawi, he is currently an Assistant Professor in Electrical and Computer Dept, Faculty of Engineerig, International Islamic University Malaysia. She received her Phd from School of Electronics and Computer Science, University of Southampton, UK, in 2012. She obtained her M. Eng in Communication and Computer Engineering from University Kebangsaan Malaysia (UKM), and her Bachelor degree from International Islamic University Malaysia (IIUM). Her current research interests include wireless communication, software defined radio, software engineering, empirical software engineering, Agile methods and software processes. She is a member of IEEE, IEEE Computer Society, a registered member for (BEM) and IEM. 